\title{
ERSTELLUNG EINES ANDROID-BASIERTEN LERNMEDIUMS ZUM THEMA ,ARBEITSWELTEN”
}

\author{
Etha Maria Sinaga \\ Ahmad Sahat Perdamean \\ Tanti Kurnia Sari
}

\begin{abstract}
AUSZUG
Das Ziel dieser Untersuchung ist, um Android- basierten Lernmediums zum Thema „Arbeitswelten” zu erstellen. In dieser Untersuchung wird die deskriptive qualitative Methode angewendet. Der Prozess der Erstellung eines Android-basierten Lernmediums zum Thema „Arbeitswelten” besteht aus Erklärung der Phasen von Richey und Klein. Darunter sind: (1) die Planungphase, (2) die Erstellungphase und (3) die Evaluationphase. Die Daten in dieser Untersuchung sind Bilder und Wörter aus dem Buch Studio d A2. Diese Untersuchung wird in der Bibliothek an der Fakultät für Sprache und Kunst an der Staatlichen Universität Medan durchgeführt. Das Thema "Arbeitswelten" besteht aus Berufsbiografien, demtabellarischen Lebenslauf, der Berufswünsche, der Grammatik ,weil" und „denn”, Nominalisierungen, Hoflichkeit, der Wortschatz des Berufs, und Übungen. Die Bewertung der Expertin über die Qualität des Ergebnisses der Erstellung eines Android-basierten Lernmediums zum Thema „Arbeitswelten” ist sehr gut.Hoffentlich kann dieses Lernmedium nützlich sein, um den Lernprozesszuerleichtern und die Lernendenkönnen Deutsch einfach und viel Spa $\beta$ lernen.
\end{abstract}

Schlüsselwörter: die Erstellung, das Lernmedium, Android

\section{EINLEITUNG}

Die Entwicklung der Technologie wächst sehr schnell, besonders im Bereich der Ausbildung. Die Ausbildung kann nicht von dem Lernprozess getrennt werden. Der Lernprozess ist ein Prozess, um Wissenschaft zu vermitteln und um ein Lernziel zu erreichen. Deshalb wird ein Medium im Unterricht braucht.

Medium ist sehr wichtig beim Unterricht, um das Interesse Studenten $\mathrm{zu}$ erhöhen. Im Lernprozess soll der Dozent eine effektive und angenehme Lernatmosphäre schaffen, damit die Studenten beim Lernen motiviert sind. Eine Möglichkeit ist dabei die Benutzung von innovativen Lernmedien. Die Verwendung der Lernmedien im Lernprozess ist sehr wichtig, denn ohne Medien ist ein Lernprozess nicht effektiv. 
Im Allgemeinen benutzen Dozent Lehrbücher und Wörterbücher im Unterricht. Basierend auf dieser Beobachtung ist die Verwendung des Lernmediums noch nicht effektiv, vor allem beim Thema „Arbeitswelten”, denn es wird nur ein Lehrbuch benutzt. Es wäre besser, wenn verschiedene Medien benutzt würden, denn der Unterricht wäre interessanter, und die Studenten bekämen viel mehr Informationen.

Sagala(in Danusaputra, 2015:5) behauptet, dass Lernressourcen, die sich nur auf das Lehrern konzentrieren, nicht genug sind, sodass ein neuer Weg gebraucht wird, um Lernmaterial zu vermitteln. Deshalb wird eine innovative, effektive, flexible und effizienze Entwicklung der Lernmedien nötig. Die Dozenten können innovative Medien im Lernprozess verwenden, um eine angemessene Lernatmosphäre schaffen.

Hardianto (in Oktiani, 2015:2) behauptet, dass die Verwendung von geeigneten und vielfältigen Medien die Motivation der Studenten im Unterricht steigern kann. Die Studenten werden aktiver und sind beim Lernen nicht gelangweilt. Eine Technologie, die heute sehr beliebt ist, ist Android. Diese Technologie kann als Lernmedium verwendet werden, um einige Probleme der Studenten beim Lernen zu lösen.

Eine Applikation ist Computerprogramm erstellt, um Menschen bei der Durchführung bestimmter Aufgaben unterstützen kann. Auf Android basierte Applikationen wurden ausgewählt, weil sie effektive, effiziente und praktische Lernmedien als dickes Buch für die Schüler darstellen, die immer und überall verwendet werden kann.

Applikationen, die auf Android basieren, haben die Vorteile, dass sie Lernmaterial in verschiedenen Formen zeigen können, wie beispiels weise in Text, Bild - oder Videoform hinzuzugefugt, macht die Lernen einfach und macht der Lernprozess angenehm. Aber die Digitale Lernmedien können nicht $100 \%$ die gedrucktene Medien übernommen werden und die Applikation kann nicht verwendet, wenn das Android nicht haben.

In dieser Untersuchung wird ein Lernmedium zum Thema „Arbeitswelten” entwickelt. Dieses Thema wurde ausgewählt, da es sehr interessant ist. Die Lehrer benutzen meist nur Lehrbücher, um dieses Thema zu unterrichten. Durch ein auf Android basierendes Lernmedium kann der Lernprozess daher interessanter gestaltet werden. 


\section{THEORETISCHE GRUNDLAGE}

\section{Der Begriff der Erstellung}

Diese Untersuchung ist eine Research and Development (R\&D) Untersuchung. Diese Untersuchungsmethoden werden verwendet, um bestimmte Produkte herzustellen und die Wirksamkeit dieser Produkte zu testen. In dieser Untersuchung wird die Entwicklungstheorie von Richey und Klein verwendet.

Richey und Klein (in Sugiyono, 2016:30) stellen fest, dass der Schwerpunkt auf dem Design und der Erstellung von Produkten liegt. Dieser Prozess kann von Anfang bis Ende analysiert werden. Im Folgenden werden die einzelnen Schritte des Entwicklungsmodells von Rickey und Klein erklärt.

\section{a. Die Planung}

In der Planungsphase wird das Design des Produkte für einen bestimmten Zweck entworfen. Die Planungsphase wird mit einer Bedarfsanalyse durch eine Untersuchung und ein Studium der Literatur durchgeführt.

\section{b. Die Herstellung}

Die zweite Phase ist die Herstellung. Dabei werden die auf Android basierenden Lernmedien erstellt. Die Herstellungumfasst alle Tätigkeiten, um das Produkt nach dem Entwurf zu erstellen.

\section{c. Die Evaluation / Bewertung}

Die dritte Phase ist die Evaluation (Bewertung). In dieser Phase wird das Produkt geprüft und bewertet. Das Ziel ist es herauszufinden, ob die Qualität des Produkts gut ist oder noch eine Verbesserung nötig ist.

\section{Der Begriff des Lernmediums}

Die Digitale Medien sind sehr wichtig im Unterricht. Taylor und Sharples (in Jorg, 2012:287) behaupten, dass die Lernmedien dienen als Sammelbegriff für alle Materialien, Medien, Inhalte, Artefakte, Dokumente, Geräte und Ähnliches dienen, die dazu verwendet werden, den Lernprozess zu gestalten. Einige Beispiele dafür sind 
Bücher, Manuskripte, digitales Material, Karten, Handbücher, Gemälde oder Computer. Cecep. K \& Bambang. S (in Fadlilah, 2015:2) sagen, dass das Lernmedium ein Mittel ist, das den Lernprozess unterstützen kann.

Es kann also zusammengefasst werden, dass die Medien ein Mittel beim Lernprozess sind, die dabei helfen können, das Lernziel $\mathrm{zu}$ erreichen und das Verständnis der Schüler zu steigern.

\section{Der Begriff der Applikation}

Nach Jogiyanto (in Permani, 2014:2) sind Applikationen die Verwendung in einem Computer, der Befehl oder die Aussage werden so angeordnet, sodass der Computer den Input in den Output verarbeiten kann. Dhanta (in Permani, 2014:2) behauptet, dass Applikationen Softwaressind, die von einer Computerfirma produziert wird, um bestimmte Aufgaben zu erledigen wie z. B. Microsoft Word, Microsoft Excel.

Nach den Erklärungen können zusammengefasst werden, dass die Applikation eine Software ist, die Verwendung im Computer hilft den Menschen die Aufgaben zu bearbeiten.

\section{Begriff das Androidsystem}

Lee ( in Kusumaningrum, 2016:18) bezeichnet Android als ein Open Source Betriebssystem für mobile Geräte, dass selbst auf einem Linux-Betriebssystem basiert. Und Nazaruddin (in Kusumaningrum, 2016:18) definiert Android ebenfalls als ein Betriebssystem für Handys, welches auf Linux basiert.

Android umfasst die Gesamtheit aller Applikation, von Betriebssystemen bis hin zur Entwicklung der Applikationen selbst. Zur Entwicklung von Androidapplikationen sind Grundlagen der Java-Programmierung notwendig. Die Entwicklungsplattform von Androidapps hat eine „Open Source - Lizenz” und ist somit kostenlos für alle zugänglich.

\section{KONZEPTUELLEN GRUNDLAGE}

Medien sind Mittel im Lernprozess. Im Allgemein benutzen Lehrer meist nur Lehrbücher als Medien und das ist nicht effektiv, denn es macht den Unterricht uninteressant. Deshalb brauchen die Lehrer neue Medien zur Verbesserung der Lernergebniss und diese können auch den Lehrern im Unterricht helfen. 
In dieser Untersuchung wird das auf Android basieren ausgewählt. Die Lernmedien, die auf Android basieren, können den Lernprozess interesanter, effektiver und praktischer gestalten, weil die Verwendung dieser Applikation sehr einfach ist. Dieses Medium kann das Interrese und die Motivation der Schüler im Lernprozess steigern.

Dieses Medium kann zum beispiel Bilder, Texte und Videos anzeigen. Außerdem kann man dieses Medium immer und überall verwenden. In dieser Untersuchung wird ein Lernmedium zum Thema „Arbeitswelten” aus dem Buch Studio D A2 in einer Androidapplikation entwickelt.

In dieser Untersuchung wird das Model von Richey und Klein verwendet, das aus drei Schritten besteht. Diese sind 1) die Planung (2) die Herstellung des Produkts und (3) die Bewertung und Verbesserung.

\section{UNTERSUCHUNGSMETODE}

Diese Untersuchung ist eine Entwicklungsuntersuchung. Diese Untersuchung wird die deskriptive qualitative Methode benutzt. Diese Untersuchung wird die Theorie von Richey und Klein-Modell benutzt.

\section{Die Daten und Die Datenquelle}

Die Daten in dieser Untersuchung sind der Wortschatz und die Texte zum Thema „Arbeitswelten”. Die Daten werden aus dem Buch Studio D A2 und verschiedene relevante Quelle genommen. Diese Untersuchung wird in der Bibliothek in der Fakultät für Sprachen und Kunst Staatliche Universität Medan durchgeführt.

Diese Untersuchung benutzt die Theorie von Rickey und Klein. Diese Theorie wurde schon in Kapitel II erklärt. In dieser Theorie gibt es drei Hauptschritte.

\section{ERGEBNIS DER UNTERSUCHUNG}

\section{Der Prozess der Erstellung}

\section{Die Planungsphase}

Dies ist die erste Phase, um ein Android-basierten Lernmediums zum Thema „Arbeitswelten” zu erstellen. In dieser Phase wird eine Beobachtung über die Verwendung des Lernmediums im Unterricht gemacht. Vor das Android-basierten Lernmedium erstellt wird, werden das Material über „Arbeitswelten” gesammelt. 
Zuerst werden die Daten zum Thema „Arbeitswelten” aus dem Buch Studio d A2 gelesen. Es gibt acht Daten, die sind: (1) Berufsbiografien, (2) der Tabellarische Lebenslauf, (3) die Berufswünsche, (4) die Grammatik ,weil” und „denn”, (5) Nominalisierungen, (6) Höfflichkeit, (7) der Wortschatzdes Berufs und (8) Übungen.

\section{Das Design}

In dieser Phase wird das Konzept des Lernmediums mit der Applikation entworfen, um ein interessantesLernmedium $\mathrm{zu}$ erstellen. Alle Teilen in der Applikation werden entworfen. Das Design wird an das Thema „Arbeitswelten” angepasst, das in der Applikation integriert wird.

\section{Die Erstellungsphase}

Der Erstellungsprozess eines Android-basierten Lernmediums gliedert sich in drei Phasen, diese sind die Vorproduktion, Produktion und Postproduktion.

\section{Vorproduktion}

Diese Phase fängt mit der Vorbereitung der Materialien zur Herstellung des Lernmediums an. Die benötigen Dinge bei der Herstellung, sind der Computer/Laptop, Dreamweaver cs6, XAMPP und Android Studio, unterstützende Materialien wie Bilder, Texte und Video, die beim Lernprozess unterstützen können.

\section{Produktion}

In dieser Phase wird die Erstellung eines Android-basierten Lernmediums zum Thema „Arbeitswelten” gemacht. Unten wird die Erstellung eines Android-basierten Lernmediums zum Thema „Arbeitswelten” erklärt.

a. Die Startseite

Auf dem Cover gibt es den Title, den loading screen in der Mitte und Informationen über die Verfasserin. Der Loading screen hat eine dauer vor fünf Sekunden duration. Das Cover wird beendet, wenn der loading screen fertig ist.

b. Das Hauptmenü

Nachdem loading screen taucht das Hauptmenü auf. Im Hauptmenü gibt es fünf Menüs: die sind Hinweis, Lernen, Übungen, Video und Ausgang. Und jedes Menü hat eigene Submenüs.

\section{Das Hinweismenü}


Das erste Menü ist das Hinweismenü. Dieses Menü besteht aus drei Submenüs, das sind Vorwort,Verwendungsziel des Lernmediums und der Hinweis des Lernmediums.

\section{Das Lernenmenü}

Lernen ist das zweite Menü. In diesem Menü gibt es einige Submenüs, nämlich Berufsbiografien, der tabellarische Lebenslauf, die Berufswünsche, die Grammatik „,weil” und „denn”, Nominalisierungen, Höflichkeit, der Wortschatz des Berufs, Text Karaoke, Glossar, Literaturverzeichnis.

a. Berufsbiografien

In diesem Submenü werdenBerufsbiografien erklärt. Es gibt zwei Berufsbiografien und Redemittel. Wenn man die Berufsbiografien anklicken, will die Texte und die Bilder von Berufsbiografien auftauchen.

b. Der tabellarische Lebenslauf

In diesem Submenü werden der tabellarische Lebenslauf erklärt. In diesem Submenü gibt es einen Lebenslauf und die Defenition von Lebenslauf. Wenn man den tabellarischen Lebenslauf anklicken, werden die Texte und das Bild von Lebenslauf auftauchen.

c. Die Berufwünsche

Im Thema Berufwünsche wird der Traumberuf erklärt. In diesem Thema gibt es die Defenition und Dialoge über Traumberuf, die Folgerung von dem Dialog und Redemittel. Wenn man die Berufwünsche anklicken, will die Texte über die Berufswünsche auftauchen.

d. Die Grammatik ,,weil” und ,denn”

In diesem Submenü wird die Grammatik ,weil” und „,denn” erklärt. Es gibt Defenition und Beispielsätze mit ,weil” und „denn”. Wenn man die Grammatikanklicken, will die Texte über die Grammatik auftauchen.

e. Nominalisierungen

In diesem Submenü wirddie Nominalisierung mit dem Infinitiv der Verben und die Nominalisierung mit-ung und Beispielsätze erklärt.Wenn man die Nominalisierungen anklicken, will die Texte über der Nominalisierungen auftauchen.

f. Höflichkeit 
In diesem Submenü wird Höflichkeit erklärt. Zu dieser Materie gibt es die Defenition und Beispielsätze.Wenn man Höfflichkeit anklicken, will die Texte über Höfflichkeit auftauchen.

g. Der Wortschatz des Berufs

In diesem Submenü wird der Wortschatz des Berufs erklärt. In dieser Materie gibt es 30 Berufe mit Ton, Bildern und Beispielsätzen. Wenn man der Wortschätze des Berufs anklicken, will die Bilder und die Texte über die Wortschatz des Berufs auftauchen. Und wenn man den Ton hören möchte, kann man $\delta$ dieses Bild anklicken.

h. Textkaraoke

In diesem Submenü wird Textkaraoke gestellt. Diese Materie besteht aus einem Dialog. Man muss den Dialog hören und ergänzen. Für das Hören kann man das Bild $\delta$ drücken. Und es gibt die Antwort von dem Dialog.

i. Glossar

In diesem Submenü wird der Wortschatz des ThemasArbeitswelten benutzt und ihre Bedeutung erklärt.

j. Literaturverzeichnis

In diesem Submenü wird die Quelle der Materie und der Übungen geschrieben.

\section{Das Übungenmenü}

Das dritte Menü ist Übungen. Dieses Menü hat einige Submenüs, das sind Übung 1, Übung 2, Übung 3, Übung 4, Übung 5 und Übung 6. Die Übungen bestehen aus 2 Arten, das sind Multiple choice und Essay.

\section{Das Videosmenü}

Dieses Menü besteht aus drei Submenüs, die sind das Informatiker Video, das Frisörin Video, das Berufe Video.

\section{Das Ausgangmenü}

Ausgang ist das Ende des Menüs. Dieses Menü funktioniert um die Applikation zu schliessen.

\section{Post-produktion}

In diesem Schritt wird die Herstellung des Android-basierten Lernmediums im Playstore hochgeladet. 


\section{Die Evaluationphase}

Das ist die letzte Phase, um ein interessantes Lernmedium zu erstellen. In dieser Phase wurde schon das Android-basierte Lernmedium erstellt. Die Validierung der Applikation besteht aus zwei Teilen. Zuerst wird was von einem Designexperten wird. Nach der Validierung wird die Applikation verbessert. Danach wird die Evaluation der Wörter und der Sprache der Applikation korrigiert, was von dem Muttersprachler gemacht wird.

Der Muttersprachler hat schon die Daten korrigiert. Nach den Kommentaren der Muttersprachlers ist schön gestaltet und informativ, es gibt noch wenige Grammatikfehlen und die Applikation regt zum Lernen an. Der Designexperte hat einige Ratschläge gegeben, die Applikation ist gut und interessant.

Basierend auf der Evaluierung der Experten werden die Applikation verbessert. Das Design der Applikation ist gut mit vorhandener Unterteilung des Menüs. Allgemein ist die Qualität des Designs sehr gut, die Darstellung ist deutlich mit dem Bild. Danach werden die Applikation publiziert können.

\section{Das Ergebnis der Erstellung}

Das Design des Lernmediums ist vielfältig und kann auf unterschiedliche Weise entworfen werden. In dieser Applikation werden 39 Bilder und 6 icon verwendet. Es gibt auch drei Videos und die Sätze. Das Video über Fachinformatiker dauert circa 2:07 Minute, das Video über Friseurin dauert circa 3:49 Minute und das Video über die Berufe dauert circa 4:40 Minuten. Die Kapazität der Applikation ist 17,55 MB.

\section{Diskussion}

Basierend auf der Ergebnissen des Lernmediums mit der Applikation wurde zusammengefasst, dass die Daten in dieser Untersuchung aus dem Buch Studio D A2, dem Internet und von einigen Web sites genommen werden. In dieser Untersuchungs wird die Rickey und Klein Theorie benutzt. Diese Theorie besteht aus drei Stufen, sie sind: (1) die Planung, (2) die Erstellung, (3) die Evaluation.

In der Planungsphase wird die Applikation zuerst offline verwendet aber das kann leider nicht gemacht werden, denn die Materie, das Video und die Töne müssen durch einen Kode gefüllt werden. Besonders müssen die Videos herunterladen. Dann werden sie zur Applikation durch einen Kode erfasst. Sodass es viel Zeit braucht um 
die Applikation zu erstellen, und die Kapazität der Applikation will sehr gro $\beta$ sein, wenn die Applikation offline gemacht. Schliessen wird eine Entscheidung getroffen, um eine online Applikation zu erstellen.

Die zweite Phase ist Erstellungphase. Das Lernmaterial wird durch die Applikation entwickelt. Die Daten werden durch ein Web erfasst. Alle Daten können in das Web umgestellt werden. Es ist sehr schwer, weil die Applikation und das Web immer geöffnet werden muss. Damit die Umstellung in der Applikation gesehen werden kann. Die Ergebnis dieser Applikation wird online verwendet, denn erleichtert die Verfasserin die Daten zu erfassen, und die Kapazität ist kleines.

In der dritten Phase wird die Bewertung und Verbesserung erklärt. Der Experte hat das Lernmedium mit derApplikationanalysiert und korrigiert. Danach wird eine Validierung durchgeführt.Der Experte benotet das Lernmedium: von sieben Kategorien der Aspekte, sodass die Note des Lernmediums mit dem Applikation ist 92,85 (sehr gut).

\section{SCHLUSSFOLGERUNG}

\section{A. Die Schlussfolgerung}

Nach den Untersuchungsergebnissen fallen die Schlussfolgerungen folgendermaßen aus:

1. Der Prozess der Erstellung eines Android-basierten Lernmediums zum Thema „Arbeitswelten” besteht aus der Erklärung des Phasen von Richey und Klein. Darunter sind: (i) Die Planung, (ii) Die Erstellung, (iii) die Bewertung und Verbesserung.

2. Die Ergebnisse der Erstellung eines Android-basierten Lernmediums zum Thema „Arbeitswelten” sind:

a. Diese Untersuchung hat einige Bilder und Videos erstellt.

b. Das Android-basierte Lernmedium über „Arbeitswelten” wurde von der Experten evaluiert. Das Design wurde von einem Designexperten gemacht. Danach wird die Evaluation der Wörter und der Sprache in der Applikation korregiert, was von dem Muttersprachler gemacht wird.

Basierend auf der obigen Meinungen kann geschlossen werden, dass die Applikation gut ist und das Thema Inhaltlich sehr interessant ist und das Ziel deutlich wird. 
https://jurnal.unimed.ac.id/2012/index.php/studia/index

\section{LITERATURVERZEICHNIS}

Arsyad, Azhar. 2013. Media Pembelajaran: Edisi revisi. Jakarta: PT RajaGrafindo Persada.

Danusaputra. 2015. Pengembangan Multimedia Interaktif Berbasis Android Pada Materi Senyawa Hidrokarbon Sebagai Media Pembelajaran Kimia SMA/MA Kelas $X$.Universitas Negeri Yogyakarta. Yogyakarta. http://eprints.uny.ac.id/38518/1/SKRIPSI.pdf, wird am 8. April 2017, um 14.00 eingeladen.

Duden. 2013. Denn-weil in Deutschland. https://www.lernhelfer.de/

Schülerlexikon/deutsch/artikel/denn-weil, wird am 14. April 2017, um 19.00 eingeladen.

Fadlilah, Hervandha. 2015.Pengembangan Media Pembelajaran Pkn Berbasis LECTORA INSPIRE Pada Siswa Kelas IV SD Negeri PendulanSumbersari Moyudan Sleman Tahun Ajaran 2015/2016.Universitas PGRI.Yogyakarta http://repository.upy.ac.id/215/1/JURNAL.pdf,wird am 8. April 2017, um 14.00 eingeladen.

Grammatiktraining.2017. grammatiktraining.de/nominalverbal/nominalisierungvonverben.https://www.grammatiktraining.de/nominalverbal/nominalisier ung-von verben.html, wird am 22. September 2017, um 10.00 eingeladen.

Herman, Funk. 2013. Studio D A2. Jakarta: Katalis.

Jorg. H, Gerhard. S, Martin. W. 2012. CSCL-Kompendium 2.0: Lehr- und Handbuch zum Computerstützten Kooperativen Lernen.München. Oldenbourg Wissenschaftsverlag GmbH. wird am 7. Mai 2017, um 20.00 eingeladen.

Kusumaningrum, Triana. 2016. Pengembangan Game Edukasi Berbasis Android Untuk Belajar Kosakata Bahasa Prancis Di SMA Negeri 2 Klaten. Universitas Negeri Yogyakarta. Yogyakarta.http://eprints.uny.ac.id/4161 8/1/SKRIPSI.pdf,wird am 8. April 2017, um 09.30 eingeladen.

Leverenz. Rainer. 2016. Der Konjunktiv 2 - Wünsche.

http://www.onlinelernen.levrai.de/deutschuebungen/grammatik_5_7/29_k onjunktiv/34_konjunktiv_2_uebungen.htm, wird am 24. Agustus 2017,um 20.00 eingeladen. 
Oktiana, Gian. 2015. Pengembangan Media Pembelajaran Berbasis Android Dalam Bentuk Buku Saku Digital Untuk Mata Pelajaran Akuntansi Kompetensi Dasar Membuat Ikhtisar Siklus Akuntansi Perusahaan Jasa Di Kelas XI MAN 1 Yogyakarta Tahun Ajaran 2014/2015. Universitas Negeri Yogyakarta. Yogyakarta.http://eprints.uny.ac.id/.../1/SKRIPSI

\%20FULL\%20GIAN\%20DWI\%20\%2811403241023. Wird am 20. April 2017, um 11.00 eingeladen.

Permani, Rezha. 2014. Perancangan dan Implementasi Aplikasi Kamus Bahasa Jerman Bergambar Sebagai Media Belajar Anak Usia Dini Berbasis Android.Amikom.Yogyakarta. http://repository.amikom.ac.id/index.php /detail/5829/PERANCANGAN\%20DAN\%20IMPLEMENTASI\%20APLIKA SI\%20KAMUS\%20BAHASA\%20JERMAN\%20BERGAMBAR\%20SEBAG AI\%20MEDIA \%20BELAJAR\%20ANAK\%20USIA\%20DINI\%20BERBASI S\%20ANDROID wird am 28. Februar 2017, um 13.00 eingeladen.

Schubert. Harald.2012. Online-Aufgaben Deutsch als Fremdsprache. https://www.schubertverlag.de/aufgaben/uebungen_a2/a2_kap1_berufe.ht m, wird am 3. September 2017, um 10.00 eingeladen.

Schuberth. Gunnar.2012.Schreibübung - Nominalisierung von Verben. https://www.grammatiktraining.de/nominalverbal/nominalisierung-vonverben.html, wird am 10. September 2017, um 21.00 eingeladen.

Schubertverlag. 2017.aufgaben/uebungen_a2/a2_kap3_nebensaetze. https://www.schubertverlag.delaufgaben/uebungen_a2/a2_kap3_nebensae tze.html, wird am 10. September 2017, um 11.00 eingeladen.

Sugiyono. 2016. Metode Penelitian dan Pengembangan: Research and Development. Bandung: Alfabeta.

Suparno. Agus. 2014. 14 Upload Project ke Play Store.http://agussuparno.com/download/Xamarin-Android.pdf, wird am 18. Juni 2017, um 12.00 eingeladen.

Verlag. Hueber. 2017. Uebungen.

http://www.hueber.de/shared/uebungen/planet/fset.php?volume=\&Lection $=1 \&$ Exercise $=3 \&$ SubExercise $=1$, wird am 1. September 2017, um 20.00 eingeladen.

https://www.google.cartoon-mechanic-holding-huge-wrench-vector-ilustration31653931\&tbm. wird am 15. September 2017, um 15.30 eingeladen.

Etha Maria Sinaga is an alumni of German language study program of Unimed, Medan. 
Studia: Journal des Deutschsprogramms p-ISSN 2301-6108 e-ISSN 2654-9573

https://jurnal.unimed.ac.id/2012/index.php/studia/index 